\title{
Dynamique paysagère du Parc National Naturel de la Forêt des Pins en Haïti (1973- 2018)
}

Waselin Salomon, Yannick Useni Sikuzani, Akoua Tamia Madeleine Kouakou, Yao Sadaiou Sabas

Barima, Karl Hermane Joseph, Jean Marie Theodat \& Jan Bogaert

Waselin Salomon : Université d'Etat d'Haïti, Haïti, Campus Henri Christophe de Limonade. 1130, Rte Nationale \# 6 Limonade (Haïti). Université de Liège, Gembloux Agro-Bio Tech Unité Biodiversité et Paysage, 2 Passage des Déportés, 5030 Gembloux (Belgique). Auteur correspondant : s.waselin@yahoo.fr, w.salomon@uliege.be

Yannick Useni Sikuzani : Unité Ecologie, Restauration Ecologique et Paysage, Faculté des Sciences Agronomiques, Université de Lubumbashi, 1825, Lubumbashi (RD Congo).

Akoua Tamia Madeleine Kouakou : Université Jean Lorougnon Guédé, Unité de Formation et de Recherche Environnement, 150 Daloa (Cote d'Ivoire).

Yao Sadaiou Sabas Barima : Université Jean Lorougnon Guédé, Unité de Formation et de Recherche Environnement, 150 Daloa (Cote d'Ivoire).

Karl Hermane Joseph : Université d'Etat d'Haïti, Haïti, Campus Henri Christophe de Limonade. 1130, Rte Nationale \# 6 Limonade (Haïti). .

Jean Marie Theodat : Université d'Etat d'Haïti (Haïti), Faculté des sciences, URBATeR, URBALaB, Angle Rues Joseph et Mgr Guilloux, Port-au-Prince, Ouest (Haïti).

Jan Bogaert : Unité Biodiversité et Paysage, Université de Liège, Gembloux Agro-Bio Tech, 2 Passage des Déportés, 5030 Gembloux (Belgique).

DOI: $10.25518 / 2295-8010.1831$

\section{Résumé :}

En Haïti, la couverture forestière est en constante régression et n'excède pas 3,5\% du territoire national. Des aires protégées ont été créés pour préserver les rares massifs forestiers, dont l’Unité 2 du Parc National Naturel de la Forêt des Pins (PNN-FP2). Toutefois, les maigres ressources forestières de ce parc sont sujettes aux diverses pressions anthropiques, telles l'agriculture, l'exploitation du bois d'œuvre, l'urbanisation, etc. menant à la régression de leur superficie dans le paysage. Cette étude a évalué la dynamique spatio-temporelle de l'anthropisation des écosystèmes forestiers du PNN-FP2 à partir de quatre images Landsat datant de 1973, 1986, 1999 et 2018. L'approche cartographique combinée aux outils d'analyse de l'écologie du paysage a révélé que la couverture forestière naturelle a connu, en 45 ans (de 1973 à 2018), une dynamique régressive matérialisée par une perte de 59,63\% de sa couverture au profit des classes anthropiques (Champs et jachères, Végétation dégradée et Sol nu). La régression de la couverture forestière est sous-tendue par la dissection et la fragmentation de ses taches par opposition à la création des taches de classes anthropiques. Nos résultats justifient le besoin urgent de développer une politique de gestion intégrée, adéquate et 
Dynamique paysagère du Parc National Naturel de la Forêt des Pins en Haïti (1...

participative afin de préserver durablement les forêts du PNN-FP2.

\begin{abstract}
:
In Haiti, forest cover is in constant decline and does not exceed 3.5\% of the national territory. Parks have been created to preserve the rare forests, including Unit 2 of the Pine Forest National Natural Park (PNN-FP2). However, the meager forest resources of this park are subject to various anthropogenic pressures (agriculture, timber exploitation, urbanization, etc.) leading to the decline of their area in the landscape. This study evaluated the spatio-temporal dynamics of the anthropization of forest ecosystems in PNN-FP2 from four Landsat images dating from 1973, 1986, 1999 and 2018. The cartographic approach combined with the analysis tools of landscape ecology revealed that the natural forest cover has experienced, in 45 years (from 1973 to 2018), a regressive dynamic materialized by a loss of $59.63 \%$ of its coverage to the benefit of anthropogenic classes (Fields and fallows, Degraded vegetation and Bare land) The regression of forest cover is underpinned by the dissection and fragmentation of its spots as opposed to the creation of the anthropogenic class spots. Our results justify the urgent need to develop an integrated, adequate and participatory management policy in order to sustainably preserve the forests of PNN-FP2.
\end{abstract}

\title{
Introduction
}

La forêt figure parmi les ressources naturelles dont la préservation est l'une des préoccupations du développement durable, notamment en raison de l'intérêt des générations actuelles et futures pour ses nombreux services écosystémiques (36). Cependant, les conditions socioéconomiques dans lesquelles vivent les populations des pays du Sud les rendent tributaires des activités perturbatrices des écosystèmes forestiers (37). Par conséquent, entre 2010 et 2020, la perte de la superficie forestière mondiale a été estimée à 47 millions d'hectares (14). L'Amérique du Nord et centrale figurent parmi les régions qui enregistrent des pertes importantes de la couverture forestière touchant plus d'un million d'hectares entre 2010 et 2020 en entraînant, entre autres, une diminution de ses capacités à capter et stocker le carbone, à conserver la biodiversité et à protéger les sols de l'érosion (14).

Toutefois, très peu d'attentions est accordé à la caractérisation de la dynamique de l'occupation du sol au sein des forêts des îles des Caraïbes $(34,38)$, dont celles d'Haïti. Ainsi, Haïti, avec une couverture forestière estimée à moins de 3,5\% de sa superficie $\left(27750 \mathrm{~km}^{2}\right)$ en 2015 contre près de $50 \%$ au début du 20 ième siècle (13), reste parmi les pays les moins étudiés dans les Caraïbes, en dépit du fait qu'il enregistre une déforestation largement aiguë $(10,113)$. Pour enrayer cette déforestation, diverses instances ont préconisé la mise en place des aires protégées plus nombreuses, mieux placées, plus représentatives et avec moins d'impact humain (25). Toutefois, seulement 4 des 35 aires protégées inscrites dans la législation haïtienne (la loi du 17 Août 1955 sur les zones réservées, le décret du 18 Mars 1968 définissant les parcs nationaux, etc.) font l'objet d'une forme quelconque de gestion (29). Les autres parcs, particulièrement les écosystèmes marins, se trouvent déjà dans un état de dégradation avancée suite à l'urbanisation et à leur exploitation abusive, notamment pour la quête du bois (29).

Les ressources forestières haïtiennes sont fortement affectées par les forces naturelles et anthropiques. En effet, de nombreuses régions montagneuses d'Haïti sont soumises chaque année 
à des ouragans et à d'autres phénomènes météorologiques violents qui contribuent à la chute des arbres et à l'érosion des sols (35). Toutefois, la déforestation ne cesse d'être intensifiée suite à l'explosion démographique couplée à la pauvreté, d'autant plus que près de $90 \%$ de la population rurale vit avec moins de 2 \$ USD par jour $(9,17,27)$. La déforestation en Haïti est aussi enracinée par la faiblesse des mécanismes de gestion forestière à long terme englobant le manque d'une politique bien définie et adoptée pour la gestion des ressources naturelles associé à la complexité du régime foncier (35).

En Haïti, la Forêt des Pins a été créée par Arrêté en 1937 sous le nom de la Réserve Forestière de la Forêt des Pins et couvrant une superficie de plus de 38000 ha. En Janvier 2014, l'Arrêté présidentiel en a modifié le statut et a subdivisé la forêt en deux parties : l'unité I qui se situe à l'Est et l'unité II à l'Ouest. Cette dernière, dénommée le Parc National Naturel de la Forêt des Pins (PNN-FP2), couvre la plus grande superficie en Pinus Occidentalis Sw du pays, une espèce endémique de l'île Hispaniola. Cependant le PNN-FP2 a été, depuis des décennies, sujet aux activités anthropiques, entre autres l'exploitation exclusive concédée par l'Etat à des concessionnaires, la coupe illégale du bois, les feux de forêt, les cultures itinérantes (dont les cultures sur brulis), le pâturage et l'expansion anarchique du bâti (12).

La pauvreté, le régime foncier, le faible niveau d'éducation et de la taille (six personnes en moyenne) et le nombre élevé des ménages sont aussi des facteurs importants à la base de la déforestation au niveau de la Forêt des Pins $(13,38)$. En effet, en 2013, la population totale du PNN-FP2 a été estimée à 59300 habitants répartie au sein de 10000 ménages (17) contre 13500 habitants environ distribués en 1780 ménages en 1999 (9), ce qui amplifie la pression anthropique sur les rares ressources naturelles du PNN-FP2. Ainsi, une réduction de la couverture forestière de l'ordre de $61 \%$ a été enregistrée au niveau de la Réserve forestières de la forêt des pins (ensemble formée par les deux unités) sur la période de 1977 à 1997 contre 43\% sur la période de 1959 à 1977 (3, 27). Par conséquent, plusieurs espèces animales endémiques telles que le Juniperus ekmanii Florin sont en danger critique d'extinction suite à la perte de leur habitat (24).

Face à la dégradation de la couverture forestière, divers efforts ont été consentis par des organisations non gouvernementale, notamment Helvetas, la Direction du Développement et de la Coopération suisse (DDC) et l'État haïtien en vue de définir des stratégies de gestion efficaces du PNN-FP2 à travers la mise en place d'un plan de gestion. Toutefois, ces efforts, tout en permettant d'avancer timidement vers une gestion effective (implication des organisations locales, corps de surveillance opérationnel, etc.), ne permettent pas de mitiger toutes les menaces sur les ressources du parc du fait de la progression continue de la matrice anthropique environnante vers ce parc (27).

Malgré l'ampleur de l'anthropisation du PNN-FP2, les études existantes ont quantifié et cartographié le changement du couvert forestier dans la Réserve de la Forêt des Pins de 1956-1958 et 1977-1978 (3) ou une partie de celui-ci (1979 à 2000 ; 34) par télédétection. D’autres études ont donné un aperçu de la variation spatio-temporelle de la couverture végétale le long de la frontière Haitiano-dominicaine par une approche de l'indice de végétation par différence normalisée (NDVI en anglais; 38). La dynamique de l'occupation du sol récente du PNN-FP2 n'est pas connue et l'évaluation de son impact sur les processus écologiques qui s'y déroulent n'a fait l'objet d'aucune étude scientifique.

La présente étude cartographie et quantifie les changements opérés dans la structure du paysage du PNN-FP2 en se basant sur la télédétection, les Systèmes d’Information Géographique et les 
outils d'analyse de l'écologie du paysage afin d'éclairer sur les processus écologiques qui s'y déroulent. Cette information est cruciale pour mieux orienter les décideurs dans une perspective de durabilité de la conservation de ses ressources forestières. Nous vérifions si les perturbations anthropiques (agriculture, urbanisation, etc.), de plus en plus importantes au sein du PNN-FP2, entraînent des changements de l'occupation du sol menant à un morcellement et un isolement des taches de forêts de Pin.

\section{Matériel et méthodes}

\section{Description du site d'étude}

Etendu sur 14000 hectares, le PNN-FP2 est une aire protégée située entre $18^{\circ} 17^{\prime} 10^{\prime \prime}-18^{\circ} 23^{\prime} 30^{\prime \prime}$ de latitude Nord et $71^{\circ} 53^{\prime} 10^{\prime \prime}-72^{\circ} 04^{\prime} 22^{\prime \prime}$ de longitude Ouest (Figure 1). Le PNN-FP2 présente un relief très accidenté caractérisé par une succession de collines abruptes entrecoupées de plateaux, de montagnes escarpées, de vallées encaissées et de ravines profondes, avec une altitude oscillant entre 1500 et 2680 mètres (27). Sa végétation est du type forêt tropicale humide dont le Pinus Occidentalis Sw, endémique de l'île Hispaniola, en est l'espèce dominante. Le parc enregistre un climat tempéré chaud selon le système de classification de Köppen-Geiger caractérisé par des précipitations moyennes annuelles comprises entre 1600 et $2000 \mathrm{~mm}$ et des températures moyennes annuelles d'environ $14{ }^{\circ} \mathrm{C}$; avec une moyenne pour les maxima et minima respectivement de 18 et $9^{\circ} \mathrm{C}(19)$. Le régime des pluies connaît deux saisons pluvieuses (Avril à Juin et Août à Octobre) entrecoupées d'une sècheresse estivale entre Juillet et Août et sécheresse hivernale de Novembre à Mars (24). Les principales activités économiques développées par les riverains du PNN-FP2 sont l'agriculture, le pâturage, l'exploitation du bois pour la construction et la production de bois de chauffage/cuisson $(13,27)$. 


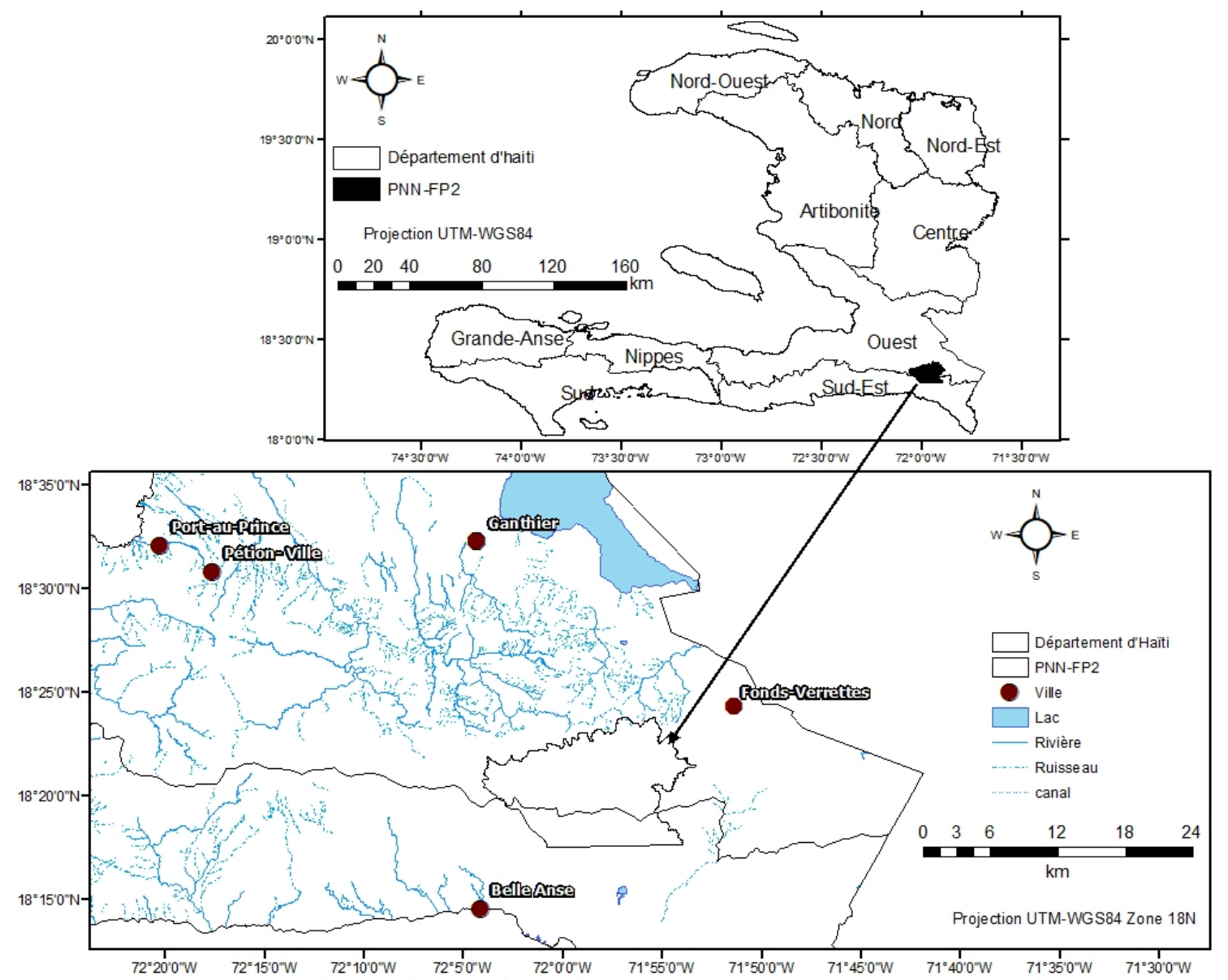

Figure 1 : Délimitation de l'Unité 2 du PNN-FP2 au niveau du département de l'ouest et du sud-est en République d'Haïti.

\section{Méthodologie}

\section{Images satellitaires et outils}

La couverture forestière étudiée a été extraite sur quatre images satellitaires téléchargées via le site http:/earthexplorer.usgsgov/. Issues du capteur Landsat, ces images datent du 28/12/1973 (capteur Multispectral Scanner System), du 20/01/1986 (capteur Thematic Mapper), du 08/01/1999 (capteur Thematic Mapper) et du 08/08/2018) (capteur Operational Land Imager) et ont une résolution spatiale de $30 \mathrm{~m}$. Le choix des capteurs retenus a été dicté par la disponibilité des images. Il est à noter que toutes les images retenues ont été acquises au cours de la saison sèche (décembre, janvier et août) afin d'obtenir une réponse spectrale plus grande des éléments paysagers (35).

En outre, les dates d'acquisition des images coïncident avec des dates clés du développement du PNN-FP2 : la période d'exploitation forestière sur le régime dictatoriale des Duvalier (en 1986), la période de reprise de la gestion de la forêt des pins par le Ministère de l'agriculture et la période de fermage de la forêt à des exploitants d'horizons différents (1999), la période d'interventions des projets de gestion plus durable (Projet d'Appui Technique pour la Protection des Parcs et Forêts (ATPPF), Helvetas Suisse) et de l'Agence Nationale des Aires Protégées (ANAP) à travers la mise en place d'une direction du parc, nécessitant l'évaluation de leurs impacts sur la réduction 
Dynamique paysagère du Parc National Naturel de la Forêt des Pins en Haïti (1...

des activités humaines (après 1999). Le logiciel ENVI 5.3 a été utilisé pour le prétraitement des images Landsat acquises et la classification de l'occupation du sol tandis que le logiciel ArcGiS 10.5.1 a servi à la mise en page cartographique.

\section{Prétraitement des images}

Un géo-référencement basé sur l'ellipsoïde de référence WGS 1984 (World Geodesic System) a été effectué selon le système de projection UTM_Zone_18N couvrant la zone d'étude. Par la suite, une correction géométrique a été appliquée sur les images de 1973, 1986 et de 1999 en se référant sur l'image Landsat de 2018. L'orthorectification de l'image de 2018 a été réalisée en se basant sur les coordonnées géographiques de 49 points de contrôle bien réparties sur toute la zone d'étude. Les autres images (1973, 1986 et de 1999) ont été orthorectifiées en référence à celle de 2018. L'analyse des changements est efficiente (22) puisque la précision géométrique du calage entre chacune des images antérieures et celle de 2018 est inférieure à 1 pixel. Par ailleurs, la correction radiométrique des images Landsat utilisées a été réalisée à travers des méthodes de régression linéaire sur la base des invariants radiométriques. Cette méthode consiste à identifier sur les images des points de repères radiométriques, caractérisés par une réflectance invariante et à déterminer les coefficients de la transformation en utilisant les statistiques de ces points (15).

\section{Classification non supervisée et collecte de données sur le terrain}

La composition colorée fausses couleurs des images Landsat isolées du PNN-FP2 a été réalisée en combinant les bandes proche-infrarouge, rouge et vert ; les deux premières étant connues comme mieux adaptées pour la discrimination de la végétation (35). La présence de végétation dense est marquée par des couleurs de plus en plus rouge alors que la présence de sol nu est traduite par des couleurs tendant vers le blanc (18). Cette composition colorée a été suivie d'une classification non supervisée sur toutes les images Landsat choisies et sur base de laquelle une interprétation visuelle de l'occupation du sol et l'identification des zones d'entraînement ont été réalisées. Huit classes de l'occupation du sol (Sol nu-bâti, zones agricoles mono ou pluri-culturales, les terres en jachère, les systèmes agroforestiers, les pâturages, les écosystèmes arbustifs et boisés dégradés, les forêts de conifère et feuillus) ont tout d'abord été créées suite à une classification automatique de l'image Landsat OLI 2018. Ce nombre de classes relativement élevé offrait la possibilité de fusionner ultérieurement des classes similaires radiométriquement.

Durant les travaux de terrain réalisés au cours du mois de novembre et de décembre 2018, un total de 520 zones de vérification a été prélevé à l'aide d'un GPS Gamin 64s ( $3 \mathrm{~m}$ de précision) dans les différentes classes d'occupations de sol du PNN-FP2 obtenues à partir de la classification non supervisée. Cet ensemble a été divisé en deux groupes de données : 345 ayant servi à la définition des zones d'entrainement sous forme de polygones de pixels sur l'image de 2018 tandis que 175 parcelles homogènes ont été utilisés pour la validation de la classification. Il est à noter que seules des parcelles homogènes et inchangées ont été choisies comme sites d'entraînement.

Le nombre de parcelles collectées au niveau de chacune des classes d'occupation du sol était fonction de sa représentativité sur l'ensemble de la zone d'étude, de sa superficie et de son intérêt pour cette étude (Tableau 1). Les zones d'entrainement sur les images de 1973, 1986 et 1999 ont été définies et matérialisées en recourant aux caractéristiques spectrales des classes d'occupation de sols obtenues de la classification finale de l'image de 2018 ; mais aussi en se basant sur l'interprétation visuelle des compositions colorées des images, standards et améliorées, sur les 
anciennes cartes thématiques de la zone d'étude ainsi que les différentes missions de terrain.

\section{Classifications supervisées et évaluation de la qualité de la classification}

L'occupation du sol a été identifiée et adaptée suivant les concepts de l'utilisation de sol du LCCS (Land Cover Classification System) de la FAO (11) afin d'assurer la cohérence de notre système de classification avec les définitions des classes d'occupation de sol utilisées par le Centre Nationale d'Informations Géospatiales (CNIGS) et celle utilisée dans le plan de gestion du Parc. D'autant plus que ce système de classification est extensible, traçable et contractile afin de le rendre compatible avec différents systèmes de classification. Il permet également de généraliser des produits régionaux plus complexes en un produit de données simplifié avec moins de classes $(10,11)$.

Par la suite, l’occupation du sol a été cartographiée sur chacune des images Landsat composites par une approche de classification supervisée en utilisant l'algorithme du maximum de vraisemblance. Cette méthode fournit à chaque pixel un indice de certitude lié à ce choix en plus de la classe à laquelle il a été affecté (22). L'occupation du sol a été définie en 4 classes de l'occupation du sol : Sol nu et bâti, Champs et jachères, Végétation dégradée, Végétation arborée dominée par le Pinus occidentalis et désignée comme étant la classe « naturelle » (Tableau 1). 
Tableau 1 : Définition des classes d'occupation du sol au sein du PNN-FP2

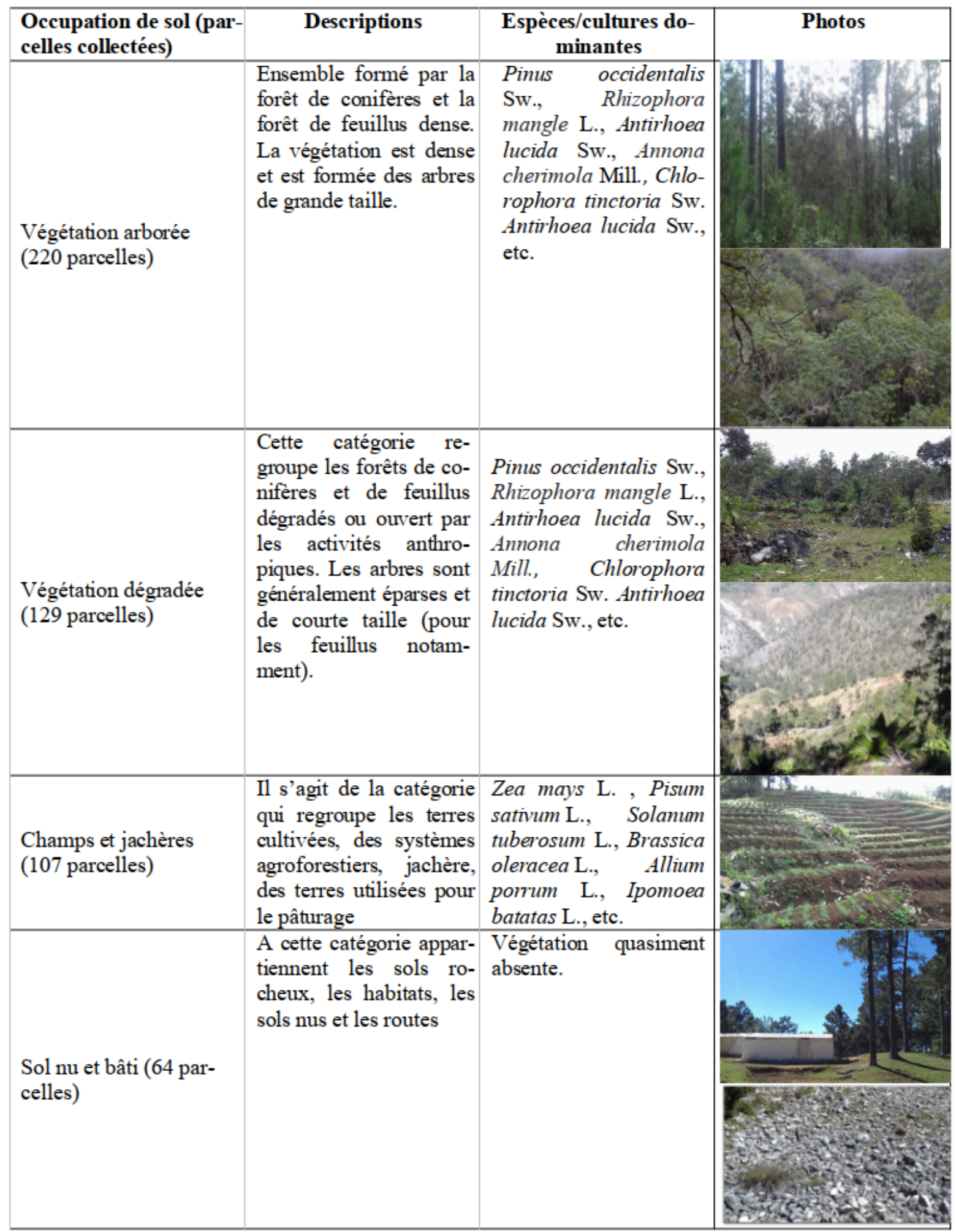

Les classifications des images obtenues ont été validées sur la base du coefficient de Kappa et de 
la précision globale, issus de la matrice de confusion. La précision de l'utilisateur correspondant au taux de pixels bien classés de la classe $i(P u(i))$ a été obtenue à partir de cette matrice suivant l'équation I (2) :

$$
\mathrm{Pu}(\mathrm{i})=\frac{M c(i)}{M_{1}}
$$

\section{(Equation I)}

$M c(i)$ correspond au nombre de pixels de la classe $i$ ayant effectivement été affectés à celle-ci après classification et $M_{1}$ la somme des pixels de la classe $i$ dans l'image. La précision du réalisateur $(\operatorname{Pr}(i))$ a été obtenue suivant l'équation II :

$$
\operatorname{Pr}(\mathrm{i})=\frac{M c(i)}{M_{2}}
$$

où $M_{2}$ représente le nombre de pixels appartenant effectivement à la classe $i$. Une valeur élevée de $\operatorname{Pr}(i)$ indique que la classe a été peu affectée par les échantillons des autres classes. À l'inverse, un taux faible indique que cette classe a souvent été choisie à la place d'autres classes (1).

Malgré quelques critiques formulées sur l'indice Kappa (30), son utilisation dans cette étude se justifie par le fait que le nombre de pixels de sites d'entrainement de chacune des classes d’occupation de sol était similaire pour les différentes dates retenues (23).

\section{Analyse de la dynamique spatiotemporelle du PNN-FP2}

Pour analyser la dynamique spatiotemporelle du PNN-FP2, des indices de structure spatiale ont été calculés. Il s'agit pour chacune des classes de l'occupation du sol, du nombre de taches ( $n$ ), de l'aire totale des taches $\left(a_{t}\right)$, de l'indice de dominance $(D)$ et de l'indice de perturbation (U). L'indice de dominance est le rapport de l'aire de la plus grande tache sur l'aire totale de la classe d'occupation du sol étudiée (37) alors que l'indice de perturbation $(U)$ est défini comme étant le rapport de l'aire cumulative des classes anthropiques (Sol nu et bâti, Champs et jachères et Végétation dégradée) et l'aire cumulative des classes naturelles (Végétation arborée) (28). Le nombre et l'aire totale des taches informent sur la fragmentation forestière matérialisée par l'augmentation du nombre suivie de la diminution de l'aire totale des taches d'une classe $(6,33)$. En écologie du paysage, ces indices sont considérés comme un compromis idéal dans la caractérisation de la configuration du paysage (6).

Les matrices de transition, réalisées par la superposition des cartes deux à deux pour chaque période, ont permis d'analyser la dynamique de conversion des aires de classes d'occupation du sol entre 1973-1986, 1986-1999 et 1999-2018. Elles permettent de ressortir l'ampleur de la conversion ou la stabilité des classes naturelles, ce que les indices de structure spatiale ne montrent pas à priori (6). Les matrices de transition permettent aussi de quantifier la conversion entre classes d'occupation du sol. 
Dynamique paysagère du Parc National Naturel de la Forêt des Pins en Haïti (1...

L'arbre de décision dichotomique (5), se basant sur l'évolution du nombre ( $n$ ) et de l'aire totale $\left(a_{t}\right)$ des taches entre deux dates a permis d'identifier les processus de transformation spatiale dominants qui sous-tendent les dynamiques observées (7) au sein des classes végétales uniquement. Cet arbre de décision reste préférable en raison de sa simplicité et sa rapidité d'exécution (7).

\section{Résultats}

\section{Classification et cartographie}

Lévaluation des résultats issus des matrices de confusion calculées pour les 4 images Landsat traitées a indiqué des valeurs de précision globale variant de 95 à $98 \%$ et celles de l'indice Kappa comprises entre $92 \%$ et $97 \%$ (Tableau 2). Ces valeurs suggèrent une bonne discrimination des classes d'occupation du sol pour chacune des images classifiées. Cependant, quelques confusions, dont les plus importantes, sont observées entre la « Végétation dégradée » et la "Végétation arborée » où 1,43\% des pixels en 1973, 4,29\% en 1999 et 1,90\% en 2018 de la "Végétation dégradée » ont été classés dans la « Végétation arborée ».

L'image de 1986 présente la meilleure classification de la classe « Végétation arborée » avec 100\% des pixels bien rangés. Des confusions remarquables ont été enregistrées aussi entre la « Végétation dégradée » et les «Champs et jachère » où 6,38\% de pixels de la "Végétation dégradée » ont été rangés en 1999 dans la classe "Champs et jachère ». La classe "Sol nu et bâti » a été parfois confondue aux « Champs et jachères » avec 4,26\% des pixels en 1973 et 2,13\% en 1986 et 1999. La "Végétation arborée » (Précision du réalisateur > 99,50\% pour toutes les dates) semble la moins affectée par les échantillons des autres classes d'occupation du sol (Tableau 2). 
Tropicultura Tropicultura 2295-8010 Volume 39 (2021) Numéro 2, 1831

Tableau 2 : Matrice de confusion, précision globale et indice de Kappa issus de la vérification des classifications supervisées des images Landsat de 1973, 1986, 1999 et 2018 du Parc National Naturel de la Forêt des Pins sur la base de l'Algorithme du maximum de vraisemblance. 
Dynamique paysagère du Parc National Naturel de la Forêt des Pins en Haïti (1...

\begin{tabular}{|c|c|c|c|c|c|c|}
\hline \multirow[b]{2}{*}{$\begin{array}{l}\text { Données de } \\
\text { référence }\end{array}$} & \multicolumn{4}{|c|}{ Données classifiées } & \multirow[b]{2}{*}{ Total } & \multirow[b]{2}{*}{$\operatorname{Pr}(\%)$} \\
\hline & $\begin{array}{l}\text { Végétation } \\
\text { arborée }\end{array}$ & $\begin{array}{l}\text { Végétation } \\
\text { dégradée }\end{array}$ & $\begin{array}{l}\text { Champs et } \\
\text { Jachères }\end{array}$ & $\begin{array}{l}\text { Sol nu et } \\
\text { bâti }\end{array}$ & & \\
\hline \multicolumn{7}{|l|}{1973} \\
\hline Végétation arborée & 98,57 & 0 & 0 & 0 & 60,00 & 100,00 \\
\hline $\begin{array}{l}\text { Végétation } \\
\text { dégradée }\end{array}$ & 1,43 & 100,00 & 0 & 0 & 16,52 & 94,74 \\
\hline Champs et Jachères & 0 & 0 & 95,74 & 0 & 13,04 & 100,00 \\
\hline Sol nu et bâti & 0 & 0 & 4,26 & 100,00 & 10,43 & 94,44 \\
\hline Total & 100,00 & 100,00 & 100,00 & 100,00 & 100,00 & \\
\hline \multirow[t]{2}{*}{$\mathrm{Pu}(\%)$} & 98,57 & 100,00 & 95,74 & 100,00 & & \\
\hline & \multicolumn{2}{|c|}{ Précision globale = 98,55\% } & \multicolumn{2}{|l|}{$K=97,5 \%$} & & \\
\hline \multicolumn{7}{|l|}{1986} \\
\hline Végétation arborée & 100,00 & 1,85 & 0 & 0 & 61,16 & 99,53 \\
\hline $\begin{array}{l}\text { Végétation } \\
\text { dégradée }\end{array}$ & 0 & 94,44 & 0 & 0 & 14,78 & 100,00 \\
\hline Champs et Jachères & 0 & 1,85 & 97,87 & 5,88 & 14,20 & 93,88 \\
\hline Sol nu et bâti & 0 & 1,85 & 2,13 & 94,12 & 9,86 & 94,12 \\
\hline Total & 100,00 & 100,00 & 100,00 & 100,00 & 100,00 & \\
\hline \multirow[t]{2}{*}{$\mathrm{Pu}(\%)$} & 100,00 & 94,44 & 97,87 & 94,12 & & \\
\hline & \multicolumn{2}{|c|}{ Précision globale = 98,26\% } & \multicolumn{2}{|l|}{$K=96,98$} & & \\
\hline \multicolumn{7}{|l|}{1999} \\
\hline Végétation arborée & 95,71 & 1,85 & 0,00 & 0,00 & 58,55 & 99,50 \\
\hline $\begin{array}{l}\text { Végétation } \\
\text { dégradée }\end{array}$ & 4,29 & 96,30 & 6,38 & 0,00 & 18,55 & 81,25 \\
\hline Champs et Jachères & 0,00 & 1,85 & 91,49 & 5,88 & 12,76 & 93,73 \\
\hline Sol nu et bâti & 0,00 & 0,00 & 2,13 & 94,12 & 10,14 & 97,14 \\
\hline
\end{tabular}




\begin{tabular}{|c|c|c|c|c|c|c|}
\hline Total & 100,00 & 100,00 & 100,00 & 100,00 & 100,00 & \\
\hline \multirow[t]{2}{*}{$\mathrm{Pu}(\%)$} & 95,71 & 96,30 & 100,00 & 91,49 & & \\
\hline & \multicolumn{2}{|c|}{ Précision globale $=95,65 \%$} & \multicolumn{2}{|c|}{$K=92,60$} & & \\
\hline \multicolumn{7}{|l|}{2018} \\
\hline Végétation arborée & 98,10 & 1,85 & 0 & 0 & 60,00 & 99,52 \\
\hline $\begin{array}{l}\text { Végétation } \\
\text { dégradée }\end{array}$ & 1,90 & 94,44 & 2,13 & 0 & 16,23 & 91,07 \\
\hline Champs et Jachères & 0 & 3,70 & 97,87 & 2,94 & 14,20 & 93,88 \\
\hline Sol nu et bâti & 0 & 0 & 0 & 97,06 & 9,57 & 100,00 \\
\hline Total & 100,00 & 100,00 & 100,00 & 100,00 & 100,00 & \\
\hline $\mathrm{Pu}(\%)$ & 98,10 & 94,44 & 97,06 & 97,87 & & \\
\hline Total & \multicolumn{2}{|c|}{ Précision globale = 97,39\% } & \multicolumn{2}{|c|}{$K=95,51 \%$} & & \\
\hline
\end{tabular}

Les cellules des matrices représentent des Pixels en pourcentage. Pu : la précision de l'utilisateur ; Pr : la précision du réalisateur.D:।

Sur les 4 cartes obtenues (Figure 2), il a été visuellement remarqué une diminution des étendues de la végétation arborée plus accentuée à l'Est du site d'étude entre 1973 et 2018. En effet, les grandes étendues de la végétation arborée qui dominaient le paysage du PNN-FP2 ont été remplacées par les classes anthropiques (Végétation dégradée, Sol nu et bâti ainsi que les Champs et jachères ; Figure 2). 

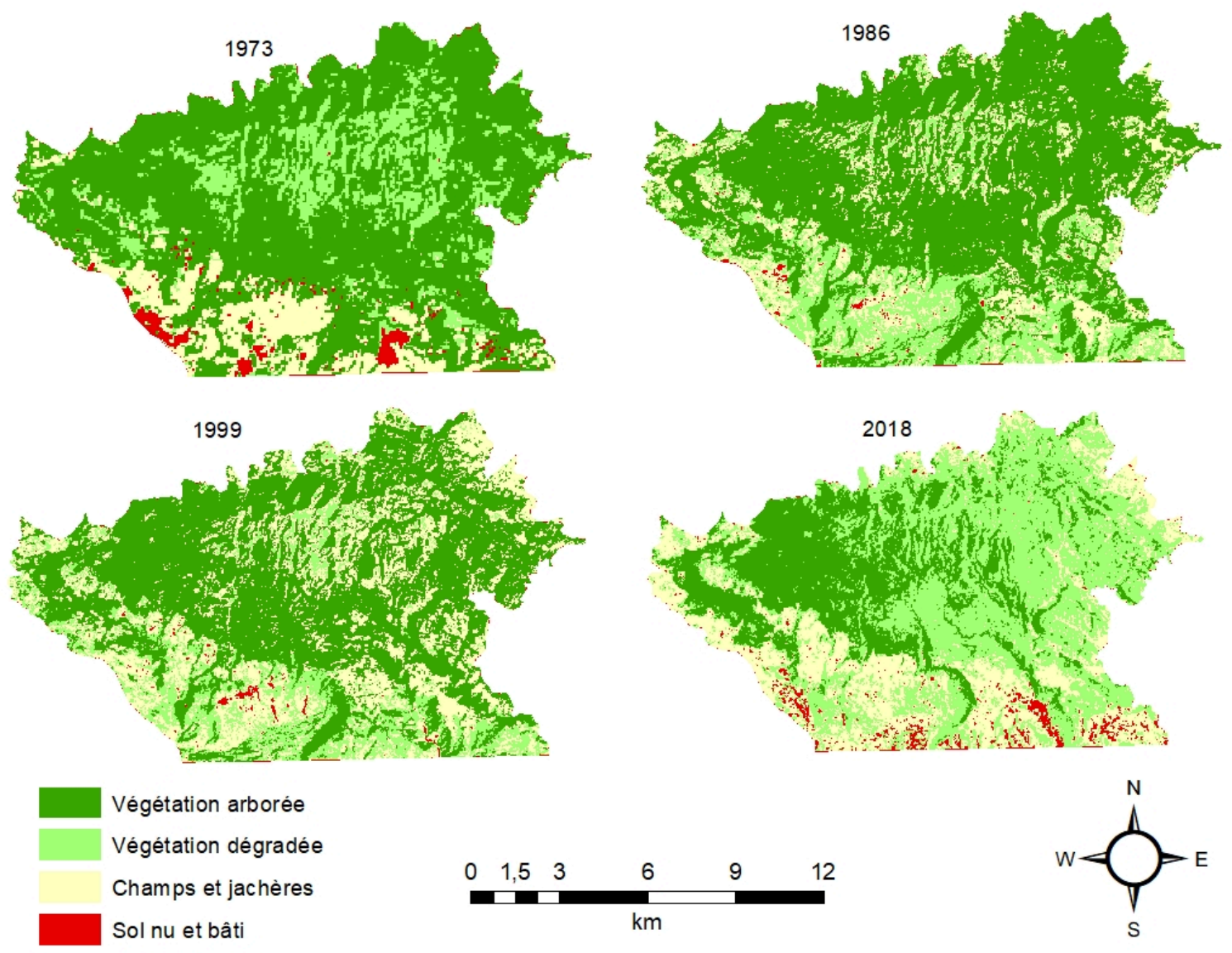

Figure 2 : Cartographie de l'occupation du sol de l’Unité 2 du Parc National Naturel de la Forêt des Pins (Haïti) sur la base de la classification supervisée d'images Landsat de 1973, 1986, 1999 et 2018.

\section{Dynamique de l'occupation du sol du PNN-FP2}

\section{Variation des proportions des classes d'occupation du sol du PNN-FP2}

Le Tableau 3 indique que la classe «Végétation arborée » qui a été la matrice du paysage durant les années 1973, 1986 et 1999 avec des proportions respectives de 68,33\%; 63,28\% et 57,33\%, a régressé de moitié de 1999 à 2018 pour couvrir27,66\% du paysage en 2018. L'évolution de cette classe indique un taux de régression annuel de 1,25\% entre 1973 et 2018 et de 2,72\% entre 1999 et 2018. En revanche, la «Végétation dégradée » a connu d'abord une légère diminution de la proportion de sa superficie, passant de 17,07\% en 1973 à 13,48\% en 1999 pour ensuite tripler dans le paysage et devenir ainsi la matrice dominante du paysage en 2018 avec une proportion de $45,23 \%$.

La superficie de «Sol nu et bâti » a diminué entre 1973 et 1986 et a ensuite été multipliée par 4 dans le paysage en passant de 0,63\% en 1986 à 2,49\% en 2018. Enfin, entre 1973 et 2018, l'étendue de la classe «Champs et jachères » a doublé dans le paysage passant de13,22\% à 24,62\%

En somme en 1973 et 2018, la tendance générale observée suite à l'analyse de la variation 
des proportions des classes d'occupation du sol (Tableau 3), a été la régression de 59,63\% de la proportion de la «végétation arborée ». En revanche, une tendance progressive des classes anthropiques a été observée à travers une expansion (entre 1973 et 2018) de l'ordre de 164,97\%, de $86,23 \%$ et de $154,58 \%$ respectivement pour les classes "Végétation dégradée », "Champs et jachères » et « Sol nu et bâti ».

\section{Transferts de l'occupation du sol}

La matrice de transition (Tableau 3) révèle qu'entre 1973-1986 et 1986-1999, plus de 50\% de la superficie de la «Végétation arborée » n'a pas connu de modification contre $24,75 \%$ entre 1999-2018 où la «Végétation arborée » a été plus fortement impactée par la déforestation. En effet, environ 75\% de la « Végétation arborée » a changé d'état en 19 ans (1999-2018), contre un peu moins de 50\% en 26 ans, (1973-1999). Ce changement entre 1999 et 2018 est observé suite à la conversion notamment de $26,85 \%$ de sa superficie en « Végétation dégradée » et de 5,43\% en «Champs et jachères ». Entre 1973 et 1986, la « Végétation arborée » a également régressé dans le paysage au profit de la «Végétation dégradée » $(7,64 \%)$, du «Sol nu et bâti » $(0,12 \%)$ et des « Champs et jachères » (8,08\%).

Quant à la "Végétation dégradée », l'analyse de la matrice de transition (Tableau 3) fait ressortir l'importance de sa progression entre 1973-2018 au détriment de la « Végétation arborée » $(31,27 \%)$ et de « Champs et jachères » (3,97\%). Entre 1973 et 1999, le développement agricole a été observé par la progression de l'étendue de la classe "Champs et jachères » au dépens de la "Végétation arborée » (8,08\% entre 1973-1986 contre 9,79\% entre 1986-1999), ainsi que de la "Végétation dégradée » (5,14\% entre 1973-1986 contre 6,65\% entre 1986-1999). La superficie des «Champs et jachères » a ensuite légèrement diminué de 1999 à 2018 au profit de la "Végétation dégradée ». Enfin, il a été remarqué une diminution de la superficie du « Sol nu et bâti » entre 1973-1986 suivie de son extension sur la période de 1986-2018 essentiellement au détriment de la "Végétation arborée » $(0,91 \%)$, de la « Végétation dégradée » $(1,125 \%)$ et des « Champs et jachères » $(1,22 \%)$.

En somme entre 1973 et 2018, la matrice de transition (Tableau 3) a révélé l'importance relative des dynamiques paysagères au sein du PNN-FP2 qui se caractérise par une stabilité des classes d'occupation du sol de $(41,02 \%$ du paysage, dont 23,36\% représente la végétation arborée) ; une dynamique de dégradation forestière $(49,02 \%$ du paysage) et enfin la dynamique de régénération (en dessous de la diagonale) de la végétation $(9,12 \%$ du paysage) 
Dynamique paysagère du Parc National Naturel de la Forêt des Pins en Haïti (1...

Tableau 3 : Matrice de transition des périodes 1973-1986, 1986-1999, 1999-2018 et 1973-2018 illustrant en pourcentage les conversions entre les différentes classes d'occupation du sol du PNN-FP2 (Haïti) sur la base des traitements des images Landsat de 1973, 1986, 1999 et 2019 classifiées (superficie totale $=140 \mathrm{~km} 2$ ). 
Tropicultura Tropicultura 2295-8010 Volume 39 (2021) Numéro 2, 1831

\begin{tabular}{|c|c|c|c|c|c|}
\hline & Végétation arborée & Végétation dégradée & Champs et jachères & Sol nu et bâti & Total \\
\hline \multicolumn{6}{|l|}{ 1973-1986 } \\
\hline Végétation arborée & 52,69 & 7,64 & 8,08 & 0,12 & 68,53 \\
\hline Végétation dégradée & 9,10 & 2,73 & 5,14 & 0,10 & 17,07 \\
\hline Champs et jachères & 0,83 & 6,21 & 5,88 & 0,30 & 13,22 \\
\hline Sol nu et bâti & 0,66 & 0,05 & 0,36 & 0,11 & 1,18 \\
\hline Total & 63,28 & 16,63 & 19,46 & 0,63 & 100,00 \\
\hline \multicolumn{6}{|l|}{ 1986-1999 } \\
\hline Végétation arborée & 51,01 & 2,39 & 9,79 & 0,09 & 63,28 \\
\hline Végétation dégradée & 2,43 & 7,38 & 6,65 & 0,17 & 16,63 \\
\hline Champs et jachères & 3,81 & 3,61 & 11,77 & 0,27 & 19,46 \\
\hline Sol nu et bâti & 0,08 & 0,10 & 0,31 & 0,14 & 0,63 \\
\hline Total & 57,33 & 13,48 & 28,52 & 0,67 & 100,00 \\
\hline \multicolumn{6}{|l|}{ 1999-2018 } \\
\hline Végétation arborée & 24,75 & 26,85 & 5,43 & 0,30 & 57,33 \\
\hline Végétation dégradée & 0,78 & 4,59 & 6,76 & 1,35 & 13,48 \\
\hline Champs et jachères & 2,13 & 13,66 & 12,13 & 0,6 & 28,52 \\
\hline Sol nu et bâti & 0,00 & 0,13 & 0,30 & 0,24 & 0,67 \\
\hline Total & 27,66 & 45,23 & 24,62 & 2,49 & 100,00 \\
\hline \multicolumn{6}{|l|}{ 1973-2018 } \\
\hline Végétation arborée & 23,36 & 31,27 & 12,99 & 0,91 & 68,53 \\
\hline Végétation dégradée & 4,08 & 9,52 & 3,35 & 0,12 & 17,07 \\
\hline Champs et jachères & 0,13 & 3,97 & 7,90 & 1,22 & 13,22 \\
\hline Sol nu et bâti & 0,09 & 0,47 & 0,38 & 0,24 & 1,18 \\
\hline Total & 27,66 & 45,23 & 24,62 & 2,49 & 100,00 \\
\hline
\end{tabular}


Dynamique paysagère du Parc National Naturel de la Forêt des Pins en Haïti $(1 \ldots$

Les chiffres en gras représentent les proportions qui n'ont pas été converties en d'autres types d'occupation du sol. Les chiffres qui sont en dessous et au-dessus de la diagonale présentent respectivement le gain et la perte de la superficie.

\section{Dynamique de la structure spatiale du PNN-FP2}

De 1973 à 1986, la « Végétation dégradée » a enregistré un accroissement du nombre et de la surface totale de ses taches, suggérant la création comme processus de transformation spatial (PTS) observé. Par contre, la «Végétation arborée » a connu la dissection $(=0,92>t=0)$ suite à l'augmentation du nombre de leurs taches parallèlement à la régression de leur aire totale (Tableau 4).

Entre 1986 et 1999, la dissection a été le PTS dominant au sein de la classe de "Végétation arborée » et de « Végétation dégradée » d'autant plus que l'augmentation du nombre des taches a été accompagnée de la régression de leur surface totale (=0; Tableau 4). De 1999 à 2018, les taches de "Végétation arborée » ont connu la fragmentation comme PTS $(=0,48<t=0$ suite à l'accroissement de leur nombre parallèlement à la diminution de leur surface totale. Sur la même période de 1999 à 2018, la création a été le PTS dominant de la « Végétation dégradée » car une augmentation du nombre et de l'aire totale de taches de ces classes a été notée (Tableau 4).

En somme entre 1973 et 2018, la végétation arborée a connu une augmentation du nombre de taches suivie d'une diminution de l'aire totale, suggérant ainsi de la fragmentation ( $t_{o b s}=$ $0,40<t=0,5$ ) comme processus de transformation spatiale dominant (Tableau 4). tandis que la « Végétation dégradée » a connu la création des taches traduite par une augmentation du nombre des taches et de leur aire totale (Tableau 2). 
Tableau 4 : Récapitulatif des indices de structure spatiale calculés pour l'identification des processus de transformation spatiale caractérisant l'évolution de la structure spatiale des classes végétales au cours des périodes 1973-1986, 1986-1999 et 1999-2018.

\begin{tabular}{|l|l|l|}
\hline & Végétation arborée & Végétation dégradée \\
\hline$n 1973$ & 70 & 467 \\
\hline$a_{t} 1973$ & 95,94 & 23,83 \\
\hline$n 1986$ & 220 & 596 \\
\hline$a_{t} 1986$ & 88,60 & 23,26 \\
\hline$n 1999$ & 514 & 1212 \\
\hline$a_{t} 1999$ & 80,27 & 18,87 \\
\hline$n 2018$ & 754 & 1727 \\
\hline$a_{t} 2018$ & 38,72 & 63,32 \\
\hline
\end{tabular}

$\mathrm{n}$ : nombre de taches; at : aire totale en $\mathrm{km}^{2}$

\section{Anthropisation du paysage}

Durant toute la période de l'étude, l'anthropisation du paysage est mise en évidence à travers la dominance de la plus grande tache de différentes classes d'occupation du sol ainsi que l'indice de perturbation (Tableau 5). Il a été noté une tendance à l'anthropisation de la « Végétation arborée » entre 1973 et 2018, matérialisée par une diminution de 25,59\% de la taille de la grande tache dans le paysage. Pendant ce temps, la taille de la plus grande tache de «Végétation dégradée » a plus que doublé de surface en passant de 32,31\% en 1973 à 74,27\% en 2018. Par conséquent, il en résulte que l'ampleur de l'anthropisation du paysage, quantifiée à travers l'indice de perturbation, a été multipliée par 6 en 45 ans passant de 0,46 en 1973 à 2,62 en 2018. La progression de l'indice de perturbation témoigne également d'une dynamique importante de diminution de la proportion des formations naturelles dans le paysage et leur remplacement par des formations anthropiques. 
Dynamique paysagère du Parc National Naturel de la Forêt des Pins en Haïti (1...

Tableau 5 : Illustration de l'anthropisation du paysage à travers l'évolution de l'indice de dominance (D) des classes végétales et de l'indice de perturbation (U) sur la base d'images Landsat de 1973, 1986, 1999 et 2018 du Parc National Naturel de la Forêt des Pins.

\begin{tabular}{|l|l|l|l|}
\hline & Végétation arborée & Végétation dégradée & \\
\hline Année & D (\%) & D (\%) & U \\
\hline 1973 & 98,16 & 32,31 & 0,46 \\
\hline 1986 & 92,19 & 37,66 & 0,58 \\
\hline 1999 & 91,35 & 15,83 & 0,74 \\
\hline 2018 & 73,05 & 74,27 & 2,62 \\
\hline
\end{tabular}

\section{Discussion}

\section{Démarches méthodologiques}

Pour quantifier les dynamiques intervenues dans le paysage forestier, les données issues de l'imagerie Landsat sont couramment utilisées, en Haïti et ailleurs $(10,34,38)$. Dans notre étude, certaines erreurs liées à l'analyse diachronique des images Landsat ont pu être limitées suite à la démarche méthodologique adoptée. En effet, les dynamiques saisonnières et interannuelles, nonquantifiables dans cette étude, ne sont pas en mesure de dominer les dynamiques décennales. Par ailleurs, les quatre images Landsat choisies au cours de la saison sèche (décembre 1973, janvier 1986, janvier 1999 et août 2018) ont contribué à diminuer d'éventuels effets saisonniers (2).

Les indices utilisés pour la mise en évidence des dynamiques spatio-temporelles de l'anthropisation du paysage du PNN-PF2 sont reconnus pour leur aptitude à aborder des paysages complexes et de les rendre quantifiables, car ils peuvent être calculés à l'échelle des taches, des classes et du paysage afin de décrire l'organisation spatiale à travers la composition, la forme et la configuration $(7 ; 35)$. Ils constituent des indicateurs fiables de l'impact humain sur la morphologie du paysage observés $(5 ; 6)$.

\section{Dynamique structurale, anthropisation et conséquences écologiques sur les écosystèmes naturels au sein du PNN-FP2}

Les résultats obtenus dans cette étude ont mis en évidence la régression des écosystèmes arborés du PNN-FP2 entre 1999 et 2018 estimée à un taux de déforestation de 51,75\%. L'intrusion multiple et répétée de l'homme dans le paysage forestier pour diverses activités dont l'agriculture et la production du charbon de bois sont à la base de la dégradation des écosystèmes forestiers (35). C'est ce qui explique l'augmentation des superficies des classes anthropiques dans le paysage du PNN-FP2 entre 1973-2018 de 164,97\%, 86,23\% et de 154,58\% respectivement pour la « Végétation dégradée », les « Champs et jachères » et le « Sol nu et bâti ». Depuis des décennies, la gestion des moteurs de déforestation par l'Etat est peu présente au niveau d'Haïti. 
À titre d'illustration, après l'échec de la Société Haitiano-américaine pour le Développement Agricole (SHADA) sur la gestion de 60500 hectares de forêt de pins dont le PNN-FP2, d'autres compagnies privées ont succédé jusqu'en 1980 avec les mêmes droits d'exploitation exclusive des ressources forestières $(13,27,35)$. Malgré la reprise de sa gestion par le Ministère de l'Agriculture des Ressources Naturelles et du Développement Rural (MARNDR) à partir de 1980, la situation de dégradation des ressources forestière n'a pas pu être redressée suffisamment. Suite à la chute de Duvalier en 1986 et à l'affaiblissement de l'autorité de l'Etat après 1986, une vague de colonisation des principales réserves naturelles du pays dont la Forêt des Pins a conduit à une dégradation presque systématique des ressources forestières (26).

Le Coup d'État de 1991 et la permanence de l'instabilité politique dans le pays ont amplifié le sabotage perpétré sur les ressources forestières au profit des cultures agricoles saisonnières (26, 27), justifiant l'expansion rapide des champs et jachères notée à travers notre étude entre 1986 et 1999 à un taux de 46,57\% (Photo 1). À ce jour, le gouvernement est peu efficace dans la gestion des ressources forestières restantes et pourrait être complice de la déforestation à certains niveaux (12). La tendance à l'évolution des classes anthropiques parallèlement à la diminution de la classe naturelle « Végétation arborée » au PNN-FP2 démontrée dans cette étude a été également observée au Parc Macaya en Haïti entre 1987 et 2000 (35), où le développement agricole accompagné de la sédentarisation des ménages agricoles à travers l'urbanisation a entrainé une diminution de la surface forestière de 45\% (14,272 ha en 1987 contre 7,861 en 2000).

Nos résultats corroborent également les résultats trouvés par Versluis et Rogan (34) dans le bassinversant de Fonds-Verrettes (Haïti) où plus de la moitié était boisée en 1979 contre près de 20\% en 2000 au profit de la classe agricole particulièrement (Photo 1), suggérant que la déforestation se produirait sur l'ensemble du parc. En outre, cette étude montre que le taux de déforestation de 2,72\% l'an au PNN-FP2 pour la période (1999-2018) est plus élevé que le taux de déforestation inférieur à 0,7\% enregistré au niveau des parcs en République Dominicaine (20).

Une telle ampleur traduit une augmentation de la pression anthropique sur la végétation arborée dominée par le Pinus occidentalis, en la convertissant en des lambeaux de forêts suite à la forte croissance démographique (13). Ce constat correspond à la situation observée dans les autres les pays d'Amérique latine et des Caraïbes où le changement de végétation ligneuse a été dominé par la déforestation suite à la pression anthropique de plus en plus forte (1).

Ainsi, les grandes taches de la végétation arborée, modérément touchées par l'anthropisation (absence de sol nu et bâti), sont confinées généralement au niveau de la zone centrale située plus au nord du PNN-FP2 sous la surveillance des vingt-huit (28) agents forestiers et de trois cadres techniques opérant dans le parc. En outre, la partie Sud du paysage du parc à l'abri de la surveillance, est dominée par des altitudes allant jusqu'à 1500 mètres, la rendant donc plus accessible à l'intrusion humaine (27).

Le faible taux de régénération de la végétation arborée (4,3\%) démontrée à la lecture de la matrice de transition entre 1973-2018 ne suffit pas pour contrecarrer la perte de superficie forestière $(45,17 \%)$ sur la même période et pourrait se justifier par quelques interventions des projets de gestion plus durable (projet ATPPF, Helvetas Suisse) et de l'Agence Nationale des Aires Protégées (ANAP) à travers la mise en place d'une direction du parc.

D’autres interventions réalisées par ces institutions pourraient également expliquer la tendance à 
Dynamique paysagère du Parc National Naturel de la Forêt des Pins en Haïti (1...

la dynamique progressive du couvert arboré, notamment : (i) la délimitation officielle du parc en 2014 et la mise en place d'un corps d'agents de surveillance forestière, (ii) le développement de certaines activités socio-économiques à impact durable pour l'accompagnement de la population dans la zone tampon, (iii) la récupération de certaines zones agricoles à des fins de reforestation, etc. En dépit de ces actions ponctuelles et limitées, le paysage du PNN-FP2 reste encore dominé par la végétation dégradée qui en constituait la nouvelle matrice paysagère en 2018.

Létude menée par Laporte (19) confirme l'augmentation constante de la surface forestière dégradée dans la région du Nord-Ouest d'Haïti. L'expansion de la végétation dégradée est un signe de perturbation des écosystèmes forestiers, provoquée notamment par une réduction du temps de jachère, une accélération des défrichements due au besoin d'étendre la superficie agricole, à la forte demande de bois pour la construction et le chauffage $(10,20)$. Ce constat est conforme à l'observation faite dans l'ensemble du pays où la végétation mixte-ligneuse a été régénérée d'environ 4\% entre 2001 et 2010 (32).

Il est à noter que la dynamique de création de la végétation dégradée à partir de la végétation arborée indique que ces milieux dégradés constitueraient des phases intermédiaires de la transformation de la forêt en une autre occupation de sol (agriculture, habitat et sol nu). Selon Mezard (24), la coupe d'arbres associée au pâturage et à l'agriculture domine parmi les facteurs à la base de la dégradation des espaces boisés au PNN-FP2. En effet, les essences forestières sont coupées puis carbonisées et vendues dans les grandes agglomérations du pays afin de répondre à plus de $70 \%$ de leur besoin énergétique (35) et les espaces ainsi défrichés sont valorisés à travers les activités agricoles.

Les facteurs contributifs au développement agricole non-conservatoire (culture sur brûlis par exemple) au sein du PNN-FP2 comprennent le manque d'incitations économiques au bénéfice des paysans et des agriculteurs (12) et une croissance démographique incontrôlée dans et autour du périmètre immédiat du parc (28), accompagné des besoins élevés en nourritures et dont la solution passe par l'extension des surfaces agricoles en périphérie du parc. Toutefois, en raison de la saturation foncière dans cette zone périphérique au parc, les ménages agricoles pénètrent au sein du PNN-FP2 pour y réaliser les activités agricoles, au détriment des espaces forestiers dont les terres arables riches en nutriments sont favorables à l'augmentation de la production agricole.

Les effets négatifs des activités agricoles sur la dégradation de la végétation des parcs est largement reconnue en Haïti $(13,20)$, ailleurs dans les Caraïbes et en Amérique Latine (4) à des ampleurs différentes. En outre, la croissance démographique est accompagnée de l'expansion incontrôlée des habitats du fait que cultiver très loin du village rend plus pénible l'activité des paysans. Par ailleurs, l'instabilité politique, l'absence d'une politique d'aménagement du territoire et les déplacements de populations de plus en plus pauvres dans ou autour de la région du parc poussent les ruraux à augmenter leurs surfaces agricoles aux dépens des espaces forestiers du PNN-FP2 (35). Ces transformations des paysages naturels en paysages anthropiques conduisent à des modifications profondes dans la structure spatiale du paysage et ont d'énormes conséquences sur les processus écologiques (érosion, inondation, etc.) et la biodiversité (31). 


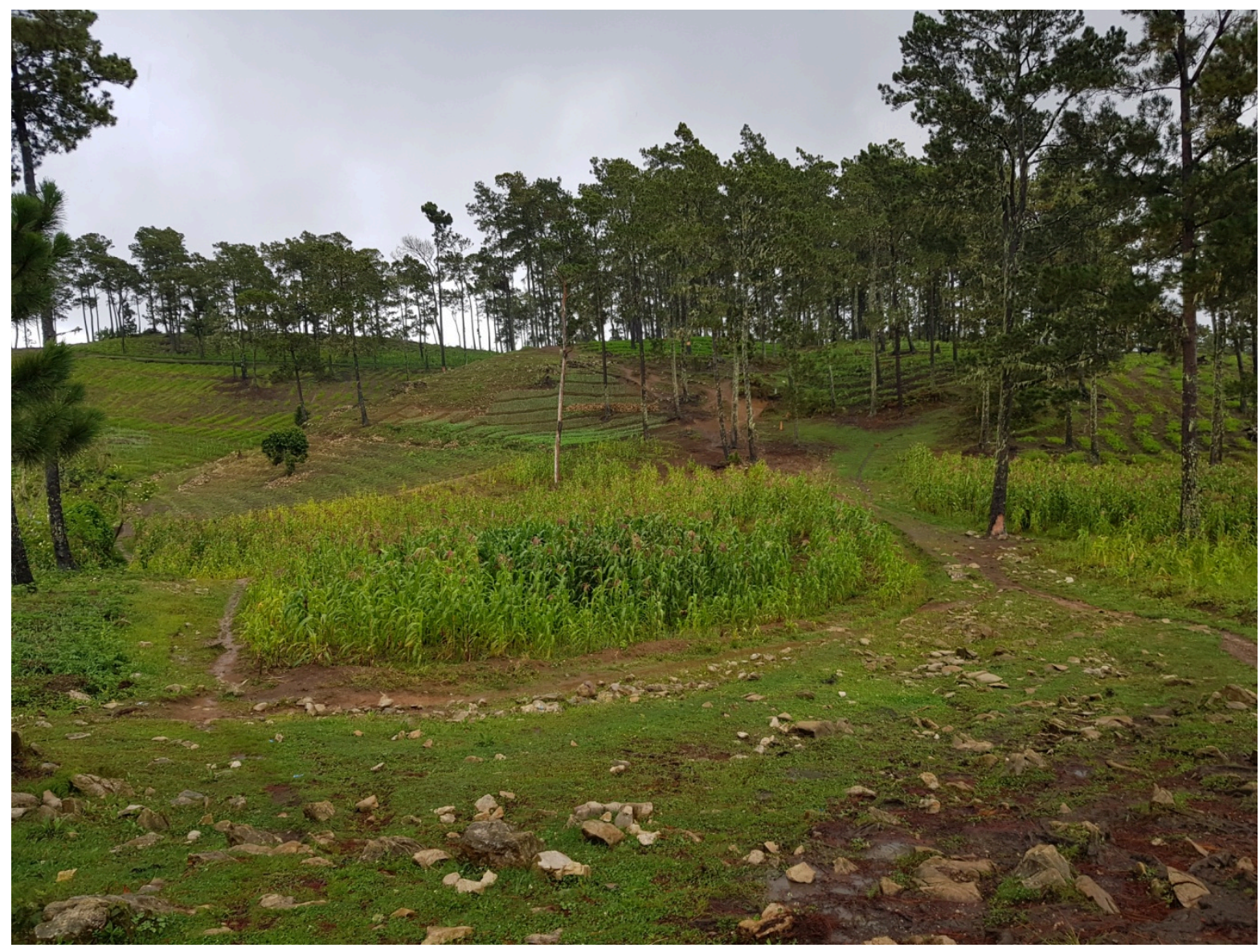

Photo 1 : Conversion de la végétation arborée dominée par le pin (Pinus occidentalis Sw.) en zone agricole, spécialement pour la culture du maïs (Zea mays L.).

Le premier plan correspond à la classe «champs et jachère », par contre le second plan, marqué par la présence d'arbres éparses, correspond à la végétation dégradée.

\section{Conclusion}

Cette étude a cartographié et quantifié les dynamiques opérées dans le paysage du PNN-FP2 à partir de l'approche cartographique associée aux outils d'analyse de l'écologie du paysage afin d'éclairer sur les processus écologiques qui s’y déroulent. Les résultats obtenus révèlent que la dynamique de la structure paysagère de ce parc est caractérisée par une régression de la couverture de la végétation arborée au profit des classes anthropiques (Végétation dégradée, Champs et jachères, Sol nu et bâti). En effet, il est démontré dans la matrice de transition que la couverture arborée dominante en 1973, 1986 et 1999 a enregistrée une régression de sa superficie au profit principalement de la Végétation dégradée qui est devenue ainsi la nouvelle matrice du paysage en 2018.

Cette diminution de la couverture forestière se produit à travers des processus de dissection et de fragmentation par opposition à la création des taches de classes anthropiques. Ceci constitue un indicateur de l'impact des activités humaines de plus en plus importantes sur la transformation continue du paysage du PNN-FP2 menant à la rupture de son équilibre écologique à travers 
Dynamique paysagère du Parc National Naturel de la Forêt des Pins en Haïti (1...

notamment le morcellement et l'isolement des taches de la Végétation arborée. Ces informations devraient permettre aux responsables politiques, aux ONG et aux aménageurs du territoire de développer une stratégie de gestion durable, effective et participative afin de limiter ainsi toutes les menaces sur les ressources forestières qui subsistent.

\section{Remerciements}

Cette étude est supportée par l'Académie de Recherche et d'Enseignement Supérieure- Commission de la Coopération au développement (ARES-CCD) et l'Agence Universitaire de la Francophonie (AUF) pour le support financier. Nous remercions la direction du PNN-FP2 pour l'appui technique et logistique mis à notre disposition durant les missions de terrain.

\section{Bibliographie}

1. Aide T.M., Clark M.L., Grau H.R., López-Carr D., Levy M.A., Redo D., \& Muñiz M., 2013, Deforestation and Reforestation of Latin A merica and the C aribbean (2001-2010). Biotropica, 45, 2, 262-271. https://dx.doi.org/10.1111/j.1744-7429.2012.00908.x

2. Barima Y.S.S., Barbier N., Bamba I., Traore D., Lejoly J. \& Bogaert > J., 2009, Dynamique paysagère en milieu de transition forêt-savane > ivoirienne. Bois For. Trop., 299, 1 , 15-25. > http://bft.cirad.fr/cd/BFT 299 15-25.pdf

3. Berry M.J. \& Musgrave K.D., 1978, Pilot management plans for pine $>$ Forest South and Central Division. Projet FAO-HAI 72/012, Port au > Prince, 68 p.

4. Blankespoor B., Dasgupta S. \& Wheeler D., 2017, Protected areas and > deforestation: new results from high-resolution panel data. Nat. > Resour. Forum, 41,55-68. DOI: 10.1111/1477-8947.12118

5. Bogaert J., Ceulemans R. \& Salvador-Van Eysenrode D., 2004, Decision > tree algorithm for detection of spatial processes in landscape > transformation. Environ. Manage., 33, 1, 62-73. DOI > 10.1007/s00267-003-0027-0

6. Bogaert J. \& Mahamane A., 2005, Écologie du paysage : cibler la > configuration et l'échelle spatiale. Ann. Sci. Agro. Bénin,, > 7, 1, 1-15. > http://hdl.handle.net/2268/ 106426

7. Bogaert J., Vranken I., Andre M., 2014, Anthropogenic effects in > landscapes: historical context and spatial pattern. In Biocultural > Landscapes Diversity, Functions and Values, Hong S.-K., Bogaert > J., Min Q. (Editor), Springer Science + Business Media Dordrescht: $>$ pp 89-112.

8. Caloz R. \& Collet C., 2001, Précis de télédétection : Traitements $>$ numériques d'images de télédétection. Volume 3, Presses de > l'Université du Québec. DOI : > 10.23071 j.ctv5j018b

9. CFET, 1999, Les Réserves d'Haïti. Manuel de Formateur en Gestion des > Zones Protégées, 37p.

10. Churches C.E., Wampler, P.J., Sun W. \& Smith A.J., 2014, Evaluation of forest cover 
estimates for Haiti using supervised classification of Landsat data. Int. J. Appl. Earth Obs. Geoinf., 30, 203-216. https://doi.org/10.1016/j.jag.2014.01.020

11. Di Gregorio A., 2005, Land cover classification system: > classification concepts and user manual: LCCS. Number 8. Food \& > Agriculture Organization, Rome, Italy, 40p

12. Direction du Développement et de la Coopération suisse (DDC), > Helvetas, Fondation Seguin, 2010. Haïti : Programme de > Préservation et de Valorisation de la Biodiversité en haute > altitude », (PVB), « Document de capitalisation de la démarche de > zonage entreprise à l’Unité 2 de la Forêt des Pins », 49 p.

13. Dolisca F., McDaniel J.M., Teeter L.D. \& Jolly C.M., 2007, Land tenure, population pressure, and deforestation in Haiti: The case of Forêt des Pins Reserve. J. For. Econ., 13, 277-289. https://doi.org/10.1016/j.jfe.2007.02.006

14. FAO. 2020. Évaluation des ressources forestières mondiales 2020 - > Principaux résultats. Rome. > https://doi.org/10.4060/ca8753fr

15. Helder D., Boncyk W., Morfitt R., 1997, Landsat TM memory effect > characterization and correction. Can. J. Remote. Sens., 23, > 4, 289-419.

16. Helvetas, 2005, Programme de Préservation et de Valorisation de la > Biodiversité en haute altitude, forêt des pins/Haïti (PVB), « > Analyse diagnostique de la problématique de gestion/valorisation $>$ des ressources naturelles et de la biodiversité dans la réserve $>$ forêt des pins" une étude de cas : l'unité ii de la réserve », > $55 \mathrm{p}$.

17. Helvetas, DDC, BUCOSEH, 2013, Situation socio-économique et > environnementale de l'Unité 2 de la Forêt des Pins. Projet de > Valorisation de la Biodiversité. Delmas 60, HTI, $109 \mathrm{p}$.

18. Kouakou A.T.M., Barima Y.S.S., Konate S., Bamba I., Kouadio J.Y. \& > Bogaert J., 2017, Gestion des forêts domaniales en période de > conflits : cas de la forêt classée du HautSassandra, Centre-Ouest > de la Côte d'Ivoire. Int. j. biol. chem. Sci., 11, 1, > 333-349. DOI: > http://dx.doi.org/10.4314/ijbcs.v11i1.26

19. Laporte J., 2013, Mapping Land Cover Change 2000 to 2013 Northwest > region, Haiti. > https://sites.tufts.edu/gis/files/2014/11/Laporte_Jessica_GIS101_FinalPoster_Fall2013.pdf

20. Lloyd J.D. \& Léon Y. M., 2019, Forest change within and outside protected 2 areas in the Dominican Republic, 2000-2016. BioRxiv, 558346. DOI : https://doi.org/10.1101/558346

21. Mama A., Bamba I., Sinsin B., Bogaert J. \& De Cannière C., 2014, > Déforestation, savanisation et développement agricole des paysages $>$ des savanes-forêts dans la zone soudano-guinéenne du Bénin. Bois > For. Trop., 322, 65-75. DOI: > https://doi.org/ 10.19182/bft2014.322.a31237

22. Mas J.F., 2000, Une revue des méthodes et des techniques de $>$ télédétection du changement. Can. J. Remote Sens., 26, > 349-362.

23. Masse A., Ducrot D. \& Marthon P., 2012, "Evaluation de > la classification supervisée par les caractéristiques de classe et $>$ de classification", Proc. SPIE 8390, Algorithmes et 
Dynamique paysagère du Parc National Naturel de la Forêt des Pins en Haïti (1...

technologies > pour l'imagerie multispectrale, hyperspectrale et ultraspectrale $>$ XVIII, 83902R (24 mai 2012); > DOI : https://doi.org/10.1117/12.919163

24. Mezard C., 2018, Caractérisation des perturbations anthropiques de $>$ la forêt des pins d'Haïti : Cas de l'unité 2. Travail de fin > d'étude en Master de spécialisation en production intégrée et $>$ préservation des ressources naturelles en milieu urbain et $>$ péri-urbain, ULiège Gembloux Agro-Bio Tech (Belgique), 79p.

25. Millennium Ecosystem Assessment, 2005, Ecosystems and human > wellbeing: World Resources Institute, Washington, DC. > https://www.millenniumassessment.org/ documents/document.358.aspx.pdf

26. Ministère de l'Environnement (MDE), 2015, Programme Aligné d'Action > National de Lutte contre la Désertification, 84 p. > https://knowledge.unccd.int/sites/default/files/ naps/Haiti-fre\%25202015.pdf

27. Ministère de l'Environnement (MDE), Direction de Développement et de > la Coopération (DDC), et Helvetas Swiss Intercooperation (HSI), > 2017, Plan de gestion 2017-2022 du Parc National Naturel de > l'Unité 2 de la Forêt des Pins, 100 p.

28. O’Neill R.V., Krummel J.R., Gardener R.H., Sugihara G., Jacksin B., > DeAngelis D.L., Milne B.T., Turner M.G., Zygmunt B., Christensen > S.W., Dale V.H. \& Grahan R.L., 1988, Indices of landscape pattern. > Landsc. Ecol., 1, 3, 153-162.

29. PNUE, Ministère de l'Environnement, UNIQ, 2010, Etat et Perspectives $>$ de l'environnement, 194 p. > http://postconflict.unep.ch/publications/Haiti/ GEO_Haiti_FR_2010.pdf

30. Pontius R.G. \& Millones M., 2011, Death to Kappa: birth of quantity > disagreement and allocation disagreement for accuracy assessment. > Int. J. Remote Sens., 32, 4407-4429. DOI : > https://doi.org/10.1080/01431161.2011.552923

31. Sordello R., Rogeon G. \& Touroult J., 2014, La fonctionnalité des > continuités écologiques - Premiers éléments de compréhension. > Service du patrimoine naturel, Muséum national

32. Tarter A., 2016, Haiti is covered with trees. EnviroSociety, $>$ Available at $>$ http://www.envirosociety.org/2016/05/haiti-is-covered-with-trees/

33. Useni S.Y., Cabala K.S., Malaisse F., Nkuku K.C., Amisi M.Y., > Bogaert J. \& Munyemba K.F., 2017, Vingt-cinq ans de monitoring de $>$ la dynamique spatiale des espaces verts en réponse à > l'urbanisation dans les communes de la ville de Lubumbashi > (HautKatanga, R.D. Congo). Tropicultura, 35, 4, 300-311. > http://hdl.handle.net/2268/227664

34. Versluis A. \& Rogan J., 2010, Mapping land-cover change in a Haitian > watershed using a combined spectral mixture analysis and > classification tree procedure. Geocarto Int., 25, 85-103. > DOI: > https://doi.org/10.1080/10106040902977584

35. Vital J. A., 2008, Land use/cover change using Remote Sensing and > Geographic Information Systems : Pic Macaya National Park, > Haiti", Master's Thesis, Michigan 
Technological > University, 2008. > https://digitalcommons.mtu.edu/etds/138

36. Wainger L.A., King D.M., Mack R.N., Price E.W. \& Maslin T., 2010, > Can the concept ecosystem services be practically applied to $>$ improve natural resource management decisions? Ecol. Econ., > 69, 978-987. DOI: > https://doi.org/10.1016/ j.ecolecon.2009.12.011

37. White J., Shao Y., Kennedy L. M., James B., 2013, Campbell Landscape > Dynamics on the Island of La Gonave, Haiti, 1990-2010. Land, > 2, 493-507. DOI: > https://doi.org/ 10.3390/land2030493

38. Wilson J.S., Brothers T.S., Marcano E.J., 2001, Remote Sensing of Spatial and Temporal Vegetation Dynamics in Hispaniola: A Comparison of Haiti and the Dominican Republic. Geocarto Int., 16, 7-18. https://doi.org/10.1080/10106040108542188

PDF généré automatiquement le 2023-04-26 13:23:50

Url de l'article : https://popups.uliege.be/2295-8010/index.php?id=1831 